\title{
Study on the Increased Probability of Detecting Adverse Drug Reactions Based on Bayes' Theorem: Evaluation of the Usefulness of Information on the Onset Timing of Adverse Drug Reactions
}

\author{
Shinji Oshima, ${ }^{a}$ Takako Enjuji, ${ }^{a}$ Akio Negishi, ${ }^{a}$ Hayato Akimoto, ${ }^{a}$ Kousuke Ohara, ${ }^{b}$ \\ Mitsuyoshi Okita, ${ }^{c}$ Sachihiko Numajiri, ${ }^{d}$ Naoko Inoue, ${ }^{d}$ Shigeru Ohshima, ${ }^{d}$ Akira Terao, ${ }^{e}$ and \\ Daisuke Kobayashi*,a \\ ${ }^{a}$ Department of Analytical Pharmaceutics and Informatics, Faculty of Pharmaceutical Sciences, Josai University; \\ 1-1 Keyakidai, Sakado, Saitama 350-0295, Japan: ${ }^{b}$ Laboratory of Drug Metabolism, Faculty of Pharmaceutical \\ Sciences, Josai International University; 1 Gumyo, Togane, Chiba 283-8555, Japan: ' Josai University Pharmacy; \\ 909-4 Shimogawara, Moroyama, Iruma-gun, Saitama 350-0435, Japan: ${ }^{d}$ Laboratory of Pharmacy Management, \\ Faculty of Pharmaceutical Sciences, Josai University; 1-1 Keyakidai, Sakado, Saitama 350-0295, Japan: and \\ ${ }^{e}$ Laboratory of Biostatistics, Faculty of Pharmaceutical Sciences, Josai University; 1-1 Keyakidai, Sakado, Saitama \\ 350-0295, Japan.
}

Received February 22, 2017; accepted May 26, 2017; advance publication released online June 3, 2017

In order to avoid adverse drug reactions (ADRs), pharmacists are reconstructing ADR-related information based on various types of data gathered from patients, and then providing this information to patients. Among the data provided to patients is the time-to-onset of ADRs after starting the medication (i.e., ADR onset timing information). However, a quantitative evaluation of the effect of onset timing information offered by pharmacists on the probability of ADRs occurring in patients receiving this information has not been reported to date. In this study, we extracted 40 ADR-drug combinations from the data in the Japanese Adverse Drug Event Report database. By applying Bayes' theorem to these combinations, we quantitatively evaluated the usefulness of onset timing information as an ADR detection predictor. As a result, when information on days after taking medication was added, 54 ADR-drug combinations showed a likelihood ratio $(L R)$ in excess of 2 . In particular, when considering the ADR-drug combination of anaphylactic shock with levofloxacin or loxoprofen, the number of days elapsed between start of medication and the onset of the ADR was 0 , which corresponded to increased likelihood ratios ( $L R s)$ of 138.7301 or 58.4516, respectively. When information from 1-7 d after starting medication was added to the combination of liver disorder and acetaminophen, the $L R$ was 11.1775. The results of this study indicate the clinical usefulness of offering information on ADR onset timing.

Key words Bayes' theorem; adverse event reporting system; adverse drug reaction; likelihood ratio; onset timing information; patient adherence instruction

One of the main tasks of a pharmacist who is engaged in dispensing drugs is to give instructions on the proper use of drugs to improve patient adherence. Article 25-2 of the Pharmacists Act requires pharmacists to provide necessary information and guidance based on pharmaceutical knowledge. Under this act, pharmacists have established a social standing as professional pharmaceutical advisers. We have recently reported the findings of an internet-based survey of 436 pharmacists and 562 patients on their opinion regarding the role of a pharmacist.") We found that a role in "responsible monitoring of patients" was closely linked to the concept of pharmacists being "advisors on the use of pharmaceuticals." Regarding the "responsible monitoring of patients," there is a large gap between pharmacists and patients in terms of how they perceive the role of a pharmacist, indicating a role discrepancy. In addition, "responsible monitoring of patients" as a subordinate concept consists of "grasping the presence/ absence of drug effectiveness," "changes in health status by drugs," and "consciousness to protect patients from adverse drug reaction (ADR)." The gap regarding role cognition between pharmacists and patients may disappear if adverse effects post administration of a medication and changes in patient health are found.
As one method to prevent the worsening of ADRs, when pharmacists are dispensing drugs they provide patients with information on the initial symptoms (ISs) of ADRs of the drug, so that patients can identify the onset of ADRs early and take the necessary action. ${ }^{2)}$ A single ADR can often have multiple ISs, and among these ISs, there will be some that are more sensitive in predicting an ADR than others. In order to increase awareness regarding ADRs, we recently reported a quantitative evaluation method using Bayes' theorem that can be used to evaluate the usefulness of different ISs of an ADR. ${ }^{3,4)}$ In one example, we looked at abnormal hepatic function, which is an ADR of terbinafine. The developed method was used to calculate likelihood ratios $(L R \mathrm{~s})$ for different ISs of abnormal hepatic function, and the calculated $L R$ values were 5.17 for fever, 2.44 for nausea, and 1.43 for a rash, indicating that fever has the highest information value and is the most useful predictor of abnormal hepatic function following treatment with terbinafine.

In addition, Ghajar et al. have reported a method that can be used to increase the diagnostic probability of whether a rash that develops while taking sulfonamide is a sulfonamideinduced ADR or not. ${ }^{5)}$ In this study, the authors analyzed five factors for prior odds using Bayes' theorem: (1) the patient's 
allergy history, (2) skin conditions, (3) the onset of a rash after taking the medication, (4) recovery after discontinuation of the treatment, and (5) the response to drug re-administration. They then calculated the posterior odds by multiplying the prior odds by the LRs of each of the above five factors, and reported that these factors increase the probability of a correct diagnosis. For example, in the case of a skin rash, if the onset of skin rash caused by sulfonamide is restricted to the beginning of treatment, onset timing information can contribute to an increase in the probability of identifying an ADR.

Accordingly, in this study, the concepts of prior probability, posterior probability, and likelihood ratio used in Bayesian statistics were applied to the identification and prevention of ADRs. Thus, when a specified ADR of a certain drug has a characteristic onset timing, confirmation of onset timing information by a pharmacist (treatment side), or the offering of this information to patients, can be expected to heighten the probability of discovering the ADR, with a certain likelihood ratio. In a similar study, without the use of likelihood ratios, safe administration of chemotherapy was successfully achieved by confirmation of ADR onset timing between the medical personnel and the patients. ${ }^{6)}$ With regard to the usefulness of likelihood ratios, Robert et al. state that "the information contributed by the test is summarized in one number corresponding to each level of test result. Additionally, likelihood ratios are particularly well suited for describing the overall odds of disease when a series of diagnostic tests is used."7) Thus, the value of offering information can be quantitatively evaluated by employing likelihood ratios., ${ }^{3,4}$ When applying Bayes' theorem to increase the ADR detection rate, the general onset probability of an ADR in the time elapsed since starting the medication is required as the prior probability, and the onset probability for a specific ADR-drug combination is required as the posterior probability. In order to obtain the general onset probability of an ADR, it is necessary to analyze many cases in order to guarantee the reliability of the analysis results. In this case, it is useful to analyze data from a large integrated ADR database or an adverse drug event reporting system database. Adverse event reporting system databases on the use of post-marketing drugs have already been established in the U.S.A., the U.K., the Netherlands, and Canada. In Japan, the Pharmaceuticals and Medical Devices Agency (PMDA) has published data on reports of spontaneous adverse events (AEs) in Japan as the Japanese Adverse Drug Event Report database (JADER). A vast amount of information on more than 300000 AEs has been collected thus far in JADER. Because the JADER database contains time information, such as the medication start date, medication end date, and $\mathrm{AE}$ date, studies performing time analyses related to the onset of AEs have been reported. ${ }^{8-10)}$

In this study, we examined the relationship between ADR onset timing information and posterior probability. According to our literature survey, no other studies have been reported that quantitatively examine the value of information provided to patients by applying Bayes' theorem to ADR onset timing information. This study quantitatively evaluated the usefulness of ADR onset timing information in an attempt to apply the concept of subjective probability to the work of community pharmacists.

\section{THEORY}

In general, the probability of whether or not a subject has a disease before undergoing an examination is expressed using the ratio of patients with that disease in the population, and this is defined as the prior probability. In the case of the examination result being positive and the patient being diagnosed with the disease, the probability that they really have contracted the disease is defined as the posterior probability. The usefulness of the diagnostic examination can then be evaluated, with a high posterior probability and a high ratio of posterior probability to prior probability (i.e., a high $L R$ ) corresponding to a high usefulness. Bayes' theorem is a method of obtaining the morbidity probability of a patient by multiplying the likelihood ratio of the examination result by the prior odds. In this study, we used the posterior probability and $L R$ to evaluate the usefulness of ADR onset timing information (i.e., the time-to-onset of the ADR, referred to as Nday-onsetinfo), by considering the prior probability as the probability of an ADR occurring after taking a general medication, and the diagnostic examination as the ADR onset time information.

Likelihood Ratio Test Based on Bayes' Theorem Bayes' theorem is given by Eq. 1:

$$
P(A \mid B)=\frac{P(B \mid A) P(A)}{P(B)}
$$

where $P(A)$ is the probability of event $A$ occurring, $P(B)$ is the probability of event $B$ occurring, $P(A \mid B)$ is the posterior probability, and $P(B \mid A)$ is the likelihood. Converting Eq. 1 to odds by dividing Eqs. 1 by 2 gives Eq. 3 .

$$
\begin{aligned}
& P\left(A^{\prime} \mid B\right)=\frac{P\left(B \mid A^{\prime}\right) P\left(A^{\prime}\right)}{P(B)} \\
& \frac{P(A \mid B)}{P\left(A^{\prime} \mid B\right)}=\frac{P(B \mid A) P(A)}{P\left(B \mid A^{\prime}\right) P\left(A^{\prime}\right)}
\end{aligned}
$$

where $A^{\prime}$ is what is not event $A$.

Equation 3 can be expressed as Eq. 4 where $A$ is the event that the patient has a disease (denoted disease + ) and $B$ is the event of a positive test result (denoted test + ).

$$
\frac{P(\text { disease }+\mid \text { test }+)}{P(\text { disease }-\mid \text { test }+)}=\frac{P(\text { test }+\mid \text { disease }+) P(\text { disease }+)}{P(\text { test }+\mid \text { disease }-) P(\text { disease }-)}
$$

Equation 4 can be expressed as:

$$
\begin{aligned}
& O d_{\mathrm{pos}}=L R \times O d_{\mathrm{pri}} \\
& L R=\frac{O d_{\mathrm{pos}}}{O d_{\mathrm{pri}}}
\end{aligned}
$$

where $L R$ is the likelihood ratio, $O d_{\text {pri }}$ is the prior odds, and $O d_{\text {pos }}$ is the posterior odds.

Further, the two-by-two contingency table of the survey data for obtaining Eq. 5 is shown in Table 1. From Eq. 5, the

Table 1. A Two-by-Two Contingency Table for Calculating the Likelihood Ratio

\begin{tabular}{lccc}
\hline \hline & Test-positive & Test-negative & Total \\
\hline Disease & $a$ & $b$ & $m$ \\
Normal & $c$ & $d$ & $n$ \\
Total & $a+c$ & $b+d$ & $m+n$ \\
\hline
\end{tabular}


likelihood ratio is given by the equation below:

$$
\begin{aligned}
\text { when } a & \ll b \text {, and } c \ll d, \\
\qquad L R & =\frac{P(\text { test }+\mid \text { disease }+)}{P(\text { test }+\mid \text { disease }-)}=\frac{a / m}{c / n}=\frac{a n}{c m}
\end{aligned}
$$

where Eq. 6 is a risk ratio $(R R)$ and therefore the risk ratio estimation method can be used. The risk ratio distribution can be normalized by $\log$ transformation. ${ }^{11)}$

$$
\ln (R R) \sim N\left(0, V_{\ln R R}\right)
$$

This property can be converted into an $L R$ and used for estimation. The $95 \%$ confidence interval was estimated. ${ }^{12)}$

$$
V_{\ln (L R)}=\frac{1}{a}-\frac{1}{n}+\frac{1}{c}-\frac{1}{m}
$$

lower limit:

$$
\mathrm{e}^{\left(\ln (L R)-1.960 \sqrt{V_{\ln (L R)}}\right)}
$$

upper limit:

$$
\mathrm{e}^{\left(\ln (L R)+1.960 \sqrt{V_{\ln (L R)}}\right)}
$$

Calculation of the Prior and Posterior Probability The incidence of an ADR occurring in a predetermined, arbitrary period of time for all of the ADRs recorded in the JADER database was defined as the prior probability $\left(P_{\text {pri }}\right)$. In addition, for a specific drug-ADR combination, the probability of an ADR occurring during the same period as the prior probability was calculated and defined as the posterior probability $\left(P_{\text {pos }}\right)$.

Calculation of Likelihood Ratios From Eq. 5:

$$
\begin{aligned}
L R & =\frac{O d_{\mathrm{pos}}}{O d_{\mathrm{pri}}} \\
& =\frac{P_{\mathrm{pos}}\left(1-P_{\mathrm{pri}}\right)}{P_{\mathrm{pri}}\left(1-P_{\mathrm{pos}}\right)}
\end{aligned}
$$

The $L R$ can therefore be obtained by calculating the prior probability and the posterior probability.

\section{METHODS}

Data Sources The JADER database contains four types of data: the case list table ("demo"), the drug information table ("drug"), the ADR table ("reac"), and the primary disease table ("hist"). Each table is linked to "case identification numbers." The reason for drug administration registered in "drug," AE name registered in "reac," and primary disease name and complications registered in "hist" are based on the preferred terms (PTs) listed in ICH Medical Dictionary for Regulatory Activities/Japanese version (MedDRA/J). The version at the time of aggregation was unified in version 16.0. For the analysis in this study, "demo," "drug" and "reac" from April 2004 to June 2015 were downloaded from the PMDA website (http://www.info.pmda.go.jp/fukusayoudb/ CsvDownload.jsp). Each downloaded table was concatenated using identification numbers to create a data frame. The data structure of the JADER database is in compliance with international safety reporting guidance, ICH E2B.
Data Cleaning Because the data reported to the JADER database includes duplicate and missing data, we cleaned the data frame. ${ }^{13,14)}$ First, "gender," "age," "weight," and "height" in "demo"; "drug name (general name)," "medication start date," and "medication end date" in "drug"; and "AE" and "AE date" in "reac" were all deleted as duplicate reports of the same cases. Next, we deleted reported cases where the "medication start date," "medication end date," or "AE onset date" data was missing. In this study, we wanted to estimate how much a pharmacist could improve the detection rate of ADRs when giving medication instructions in a community pharmacy. Hence, we extracted the cases in which the drugs involved were suspected of causing ADRs and were orally administered.

Targeted Combinations of ADRs and Drugs For convenience, we extracted the top ten ADRs from the obtained cleaned dataset. In addition, we extracted the top 10 types of drug for each of the top ten ADRs (i.e., 100 types of ADR-drug combination in total). Among the 100 types of ADR-drug data extracted, those with a number of reported cases less than 100 were excluded from the calculation. Furthermore, in combinations where a specific drug had a high rate of concomitant use among the tested ADRs-drug, the effect of the concomitant drug on the ADR could not be ignored. For example, if there 100 reported cases of ADR-A occurring with drug-B, but in 50 of those cases drug- $\mathrm{C}$ was concomitantly used alongside drug-B, it can be inferred that drug-C has some effect on the onset of ADR-A. Therefore, for convenience, we excluded ADR-drug combinations involving specific drugs whose rate of concomitant use exceeded $20 \%$ from the calculation.

Time-to-Onset Analysis Utilizing LRs We calculated the number of days to ADR onset from the "medication start date" and the "AE onset date" in "reac" using Eq. 8.

$$
\begin{aligned}
& \text { (Number of days to ADR onset) } \\
& \quad=(\text { AE onset date })-(\text { Medication start date })
\end{aligned}
$$

The ADR onset timing was calculated in intervals of general prescription days in Japan. The number of days to ADR onset was divided into nine predefined periods: (1) 0 (first day taking medication), (2) 1 to $7 \mathrm{~d}$, (3) 8 to $14 \mathrm{~d}$, (4) 15 to $30 \mathrm{~d}$, (5) 31 to $60 \mathrm{~d},(6) 61$ to $90 \mathrm{~d},(7) 91$ to $180 \mathrm{~d},(8) 181$ to $365 \mathrm{~d}$, and (9) $366 \mathrm{~d}$ or more. For each period, $L R$ was calculated from $P_{\text {pos }}$ $\left(P_{\text {pos } 0}, P_{\text {pos1-7 }}, P_{\text {pos8-14 }}, P_{\text {pos 15-30 }}, P_{\text {pos31-60 }}, P_{\text {pos61-90 }}, P_{\text {pos } 91-180}\right.$, $P_{\text {posi81-365 }}$ and $\left.P_{\text {pos366+ }}\right)$ and $P_{\text {pri }}\left(P_{\text {pri0 } 0}, P_{\text {pril-7 }}, P_{\text {pri8-14 }}, P_{\text {pril5-30 }}\right.$, $\left.P_{\text {pri31-60 }}, P_{\text {pri61-90 }}, P_{\text {pri91-180 }}, P_{\text {pri181-365 }}, P_{\text {pri366+ }}\right)$. We used the R Projects for Statistical Computing software package ( $\mathrm{R}$ version 3.2.1, R Foundation for Statistical Computing, Vienna, Austria) for data processing. ${ }^{15)}$

\section{RESULTS AND DISCUSSION}

ADR-Drug Combinations During data cleaning, the total number of reported cases decreased from 349375 to 115718 (number of drug-ADR combinations: 357497). Table 2 shows the 100 types of drug-ADR combinations extracted from the 115718 cases. The number of cases of these 100 combinations was 19493, which was equivalent to $16.85 \%$ $(19493 / 115718)$ of the cleaned dataset. In Table $2, \star$ represents a drug-ADR combination in which the rate of concomitant 
Table 2. The 100 ADR-Drug Combinations Included in This Study

\begin{tabular}{|c|c|c|c|c|}
\hline ID & ADR & Drug & ADR-drug abbreviation & Case number \\
\hline 1 & Interstitial lung disease & Gefitinib & Il-Ge & 783 \\
\hline 2 & Interstitial lung disease & Erlotinib hydrochloride & Il-Er & 679 \\
\hline 3 & Interstitial lung disease & Methotrexate & Il-Me & 545 \\
\hline 4 & Interstitial lung disease & Tegafur $\cdot$ Gimeracil $\cdot$ Oteracil potassium & Il-Tg & 403 \\
\hline 5 & Interstitial lung disease & Everolimus & Il-Ev & 348 \\
\hline 6 & Interstitial lung disease & Amiodarone hydrochloride & Il-Am & 230 \\
\hline$\star 7$ & Interstitial lung disease & Ribavirin & & 205 \\
\hline 8 & Interstitial lung disease & Loxoprofen sodium hydrate & Il-Lo & 110 \\
\hline 9 & Interstitial lung disease & Imatinib mesylate & Il-Im & 113 \\
\hline$\star 10$ & Interstitial lung disease & Prednisolone & & 84 \\
\hline 11 & Hepatic function abnormal & Sorafenib tosylate & Hf-So & 433 \\
\hline 12 & Hepatic function abnormal & Terbinafine hydrochloride & $\mathrm{Hf}-\mathrm{Tb}$ & 243 \\
\hline 13 & Hepatic function abnormal & Ticlopidine hydrochloride & Hf-Ti & 191 \\
\hline 14 & Hepatic function abnormal & Loxoprofen sodium hydrate & Hf-Lo & 180 \\
\hline$\star 15$ & Hepatic function abnormal & Tegafur $\cdot$ Uracil & & 159 \\
\hline 16 & Hepatic function abnormal & Gefitinib & Hf-Ge & 137 \\
\hline 17 & Hepatic function abnormal & Atorvastatin calcium hydrate & Hf-At & 132 \\
\hline 18 & Hepatic function abnormal & Carbamazepine & $\mathrm{Hf}-\mathrm{Ca}$ & 130 \\
\hline 19 & Hepatic function abnormal & Fluvastatin sodium & Hf-Fl & 117 \\
\hline 20 & Hepatic function abnormal & Lansoprazole & Hf-La & 103 \\
\hline$\star 21$ & Platelet count decreased & Ribavirin & & 486 \\
\hline 22 & Platelet count decreased & Sunitinib malate & $\mathrm{Pc}-\mathrm{Su}$ & 531 \\
\hline$\star 23$ & Platelet count decreased & Simeprevir sodium & & 354 \\
\hline$\star 24$ & Platelet count decreased & Tegafur $\cdot$ Gimeracil $\cdot$ Oteracil potassium & & 369 \\
\hline$\star 25$ & Platelet count decreased & Lenalidomide hydrate & & 252 \\
\hline 26 & Platelet count decreased & Dasatinib hydrate & Pc-Da & 215 \\
\hline 27 & Platelet count decreased & Sorafenib tosylate & Pc-So & 166 \\
\hline 28 & Platelet count decreased & Everolimus & Pc-Ev & 154 \\
\hline$\star 29$ & Platelet count decreased & Dexamethasone & & 137 \\
\hline 30 & Platelet count decreased & Temozolomide & $\mathrm{Pc}-\mathrm{Tz}$ & 122 \\
\hline 31 & Anaphylactic shock & Levofloxacin hydrate & As-Le & 139 \\
\hline 32 & Anaphylactic shock & Loxoprofen sodium hydrate & As-Lo & 100 \\
\hline$※ 33$ & Anaphylactic shock & Garenoxacin mesilate hydrate & & 96 \\
\hline$※ 34$ & Anaphylactic shock & Cefcapene pivoxil hydrochloride hydrate & & 53 \\
\hline$※ 35$ & Anaphylactic shock & Diclofenac sodium & & 44 \\
\hline$※ 36$ & Anaphylactic shock & Eperisone hydrochloride & & 44 \\
\hline$※ 37$ & Anaphylactic shock & Tosufloxacin tosylate hydrate & & 40 \\
\hline$※ 38$ & Anaphylactic shock & Moxifloxacin hydrochloride & & 40 \\
\hline$※ 39$ & Anaphylactic shock & Common cold remedy (over-the-counter drugs) & & 34 \\
\hline$※ 40$ & Anaphylactic shock & Analgesic anti-inflammatory drugs (over-the-counter drugs) & & 32 \\
\hline$\star 41$ & White blood cell count decreased & Ribavirin & & 557 \\
\hline$\star 42$ & White blood cell count decreased & Simeprevir sodium & & 257 \\
\hline$\star 43$ & White blood cell count decreased & Tegafur $\cdot$ Gimeracil $\cdot$ Oteracil potassium & & 310 \\
\hline$\star 44$ & White blood cell count decreased & Telaprevir & & 268 \\
\hline 45 & White blood cell count decreased & Dasatinib hydrate & Wb-Da & 171 \\
\hline 46 & White blood cell count decreased & Sunitinib malate & $\mathrm{Wb}-\mathrm{Su}$ & 152 \\
\hline 47 & White blood cell count decreased & Temozolomide & $\mathrm{Wb}-\mathrm{Tz}$ & 111 \\
\hline$※ 48$ & White blood cell count decreased & Valganciclovir hydrochloride & & 96 \\
\hline$※ 49$ & White blood cell count decreased & Prednisolone & & 79 \\
\hline$※ 50$ & White blood cell count decreased & Procarbazine hydrochloride & & 88 \\
\hline 51 & Pyrexia & Lamotrigine & Py-Lm & 282 \\
\hline$\star 52$ & Pyrexia & Ribavirin & & 226 \\
\hline 53 & Pyrexia & Sorafenib tosylate & Py-So & 111 \\
\hline 54 & Pyrexia & Carbamazepine & $\mathrm{Py}-\mathrm{Ca}$ & 104 \\
\hline$\star 55$ & Pyrexia & Simeprevir sodium & & 94 \\
\hline$※ 56$ & Pyrexia & Tegafur $\cdot$ Gimeracil $\cdot$ Oteracil potassium & & 86 \\
\hline$※ 57$ & Pyrexia & Sunitinib malate & & 94 \\
\hline$※ 58$ & Pyrexia & Mesalazine & & 89 \\
\hline$※ 59$ & Pyrexia & Live Attenuated Human Rotavirus Vaccine, Oral & & 82 \\
\hline$※ 60$ & Pyrexia & Loxoprofen sodium hydrate & & 61 \\
\hline
\end{tabular}


Table 2. Continued

\begin{tabular}{|c|c|c|c|c|}
\hline ID & ADR & Drug & ADR-drug abbreviation & Case number \\
\hline$\star 61$ & Neutrophil count decreased & Ribavirin & & 561 \\
\hline$\star 62$ & Neutrophil count decreased & Tegafur $\cdot$ Gimeracil $\cdot$ Oteracil potassium & & 306 \\
\hline$\star 63$ & Neutrophil count decreased & Lenalidomide hydrate & & 259 \\
\hline$\star 64$ & Neutrophil count decreased & Telaprevir & & 194 \\
\hline$\star 65$ & Neutrophil count decreased & Simeprevir sodium & & 152 \\
\hline 66 & Neutrophil count decreased & Dasatinib hydrate & Nc-Da & 143 \\
\hline$\star 67$ & Neutrophil count decreased & Dexamethasone & & 141 \\
\hline 68 & Neutrophil count decreased & Sunitinib malate & $\mathrm{Nc}-\mathrm{Su}$ & 103 \\
\hline$※ 69$ & Neutrophil count decreased & Prednisolone & & 72 \\
\hline$※ 70$ & Neutrophil count decreased & Temozolomide & & 72 \\
\hline 71 & Liver disorder & Terbinafine hydrochloride & $\mathrm{Ld}-\mathrm{Tb}$ & 196 \\
\hline 72 & Liver disorder & Ticlopidine hydrochloride & Ld-Ti & 194 \\
\hline 73 & Liver disorder & Loxoprofen sodium hydrate & Ld-Lo & 136 \\
\hline 74 & Liver disorder & Sorafenib tosylate & Ld-So & 100 \\
\hline 75 & Liver disorder & Acetaminophen & Ld-Ac & 102 \\
\hline$※ 76$ & Liver disorder & Carbamazepine & & 94 \\
\hline$※ 77$ & Liver disorder & Levofloxacin hydrate & & 89 \\
\hline$※ 78$ & Liver disorder & Atorvastatin calcium hydrate & & 83 \\
\hline$※ 79$ & Liver disorder & Fluvastatin sodium & & 87 \\
\hline$※ 80$ & Liver disorder & Cefcapene pivoxil hydrochloride hydrate & & 80 \\
\hline$\star 81$ & Anemia & Ribavirin & & 1321 \\
\hline$\star 82$ & Anemia & Teraprevir & & 722 \\
\hline$\star 83$ & Anemia & Simeprevir sodium & & 251 \\
\hline$\star 84$ & Anemia & Lenalidomide hydrate & & 186 \\
\hline 85 & Anemia & Dasatinib hydrate & An-Da & 167 \\
\hline$\star 86$ & Anemia & Tegafur $\cdot$ Gimeracil $\cdot$ Oteracil potassium & & 147 \\
\hline 87 & Anemia & Everolimus & An-Ev & 122 \\
\hline$\star 88$ & Anemia & Dexamethasone & & 113 \\
\hline$※ 89$ & Anemia & Sorafenib tosylate & & 83 \\
\hline$※ 90$ & Anemia & Sunitinib malate & & 87 \\
\hline$\star 91$ & Pneumonia & Methotrexate & & 262 \\
\hline$\star 92$ & Pneumonia & Prednisolone & & 182 \\
\hline$\star 93$ & Pneumonia & Tacrolimus hydrate & & 118 \\
\hline$\star 94$ & Pneumonia & Lenalidomide hydrate & & 135 \\
\hline$\star 95$ & Pneumonia & Dexamethasone & & 115 \\
\hline 96 & Pneumonia & Tegafur $\cdot$ Gimeracil $\cdot$ Oteracil potassium & $\mathrm{Pn}-\mathrm{Tg}$ & 100 \\
\hline$※ 97$ & Pneumonia & Ciclosporin & & 54 \\
\hline$※ 98$ & Pneumonia & Everolimus & & 54 \\
\hline$※ 99$ & Pneumonia & Ribavirin & & 55 \\
\hline$※ 100$ & Pneumonia & Mycophenolate mofetil & & 30 \\
\hline
\end{tabular}

$\star$ : Combinations for which the rate of concomitant use of a certain drug exceeded $20 \%$. $※$ : Combinations for which the number of reported cases was less than 100 .

use of a certain drug exceeded $20 \%$, and $※$ represents combinations in which the number of reported cases was less than 100. There were 8598 cases (40 combinations) that were not marked with $\star$ and $※$, accounting for $7.43 \%(8598 / 115718)$ of the cleaned dataset. These cases were taken forward for the subsequent calculations.

Interpretation of $\boldsymbol{L R}$ s Table 3 shows the calculation results of the 40 types of combinations targeted for calculation. The probability of an ADR being reported during each time period $\left(P_{\mathrm{pri}}\right)$ considering all of the ADR-drug combinations was $P_{\text {prio }}=0.0605$ (21617/357497), $P_{\text {pri1-7 }}=0.1838(65712 / 357497), P_{\text {pri8-14 }}=0.1116(39885 / 357497)$, $P_{\text {pri15-30 }}=0.1446(51684 / 357497), P_{\text {pri31-60 }}=0.1358(48536 / 357497)$, $P_{\text {pri61-90 }}=0.0697(24900 / 357497), P_{\text {pri91-180 }}=0.1026(36673 / 357497)$, $P_{\text {pri181-365 }}=0.0815 \quad(29131 / 357497), \quad$ and $\quad P_{\text {pri366+ }}=0.1101$ (39359/357497). $L R$ was calculated from $P_{\text {pri }}$ and $P_{\text {pos }}$ in a preset period after taking the drug. As a result, it was clear that the onset timing of the same ADRs differs depending on the particular drug. For example, the timing of onset of interstitial lung disease was shown to be from $366 \mathrm{~d}$ onward after the start of treatment in the case of methotrexate and amiodarone hydrochloride, whereas in the case of gefitinib, erlotinib hydrochloride, tegafur/gimeracil/oteracil potassium, everolimus, loxoprofen sodium hydrate, and imatinib mesylate, interstitial lung disease occurs as an ADR within $90 \mathrm{~d}$ of starting treatment with the drug.

Three ADR-drug-onset timing (A-D-O) combinations showed an $L R$ of more than 10. In these cases, onset timing information resulted in meaningful changes in the probability of identifying the occurrence of the ADR, which is considered to have considerable clinical value., ${ }^{7,16}$ In all, there were 51 A-D-O combinations with an $L R$ greater than 2 but less than 10. In these cases, ADR onset timing information could well have certain clinical value in terms of patient awareness of 


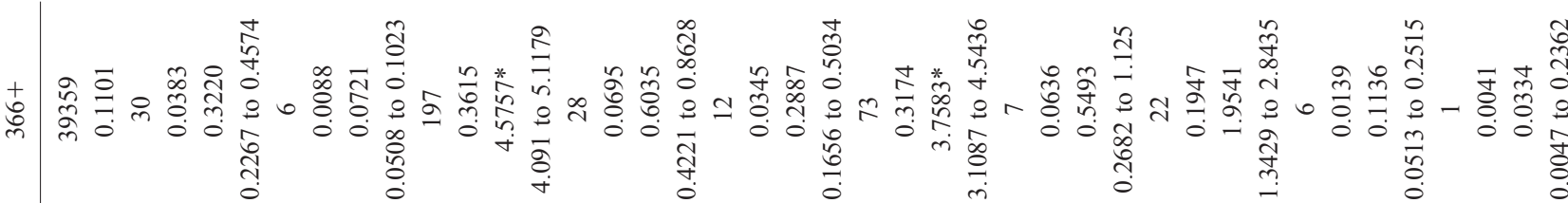

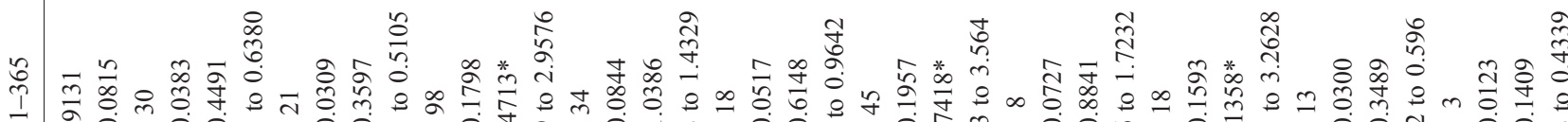

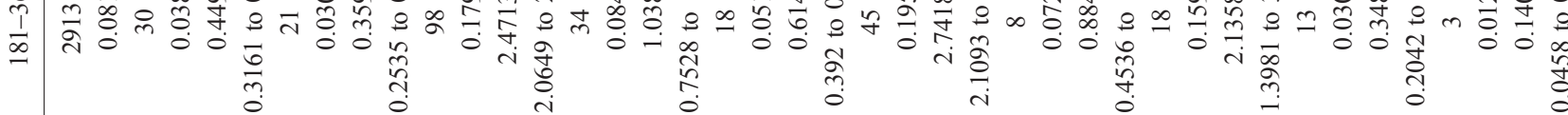

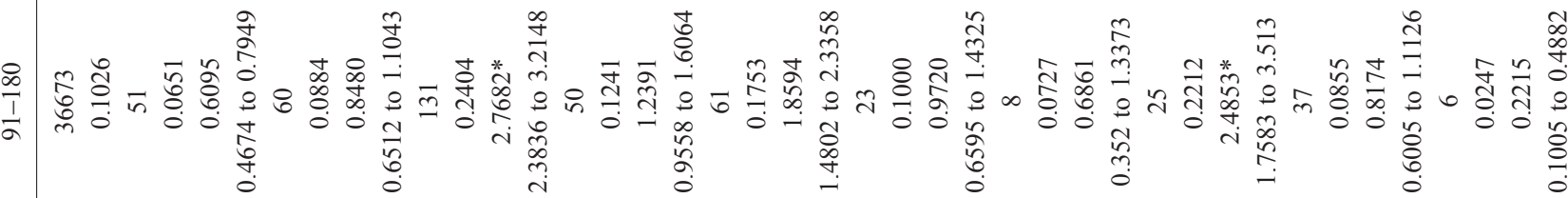

\&

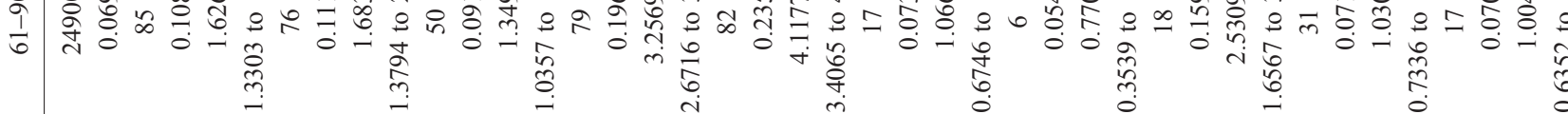

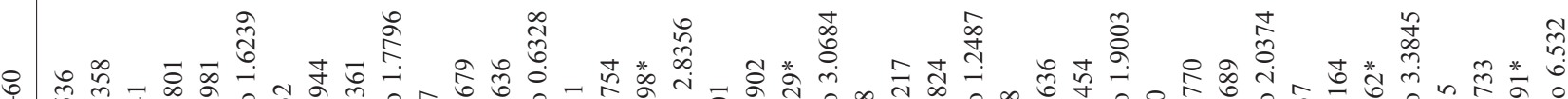

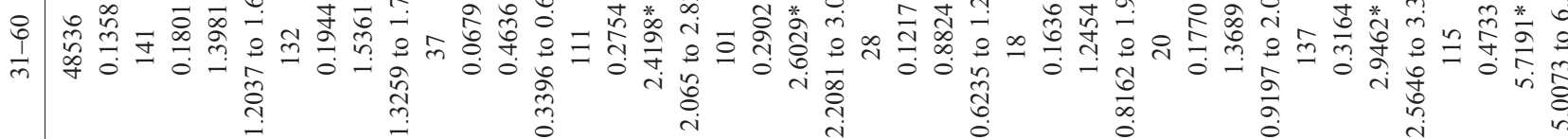

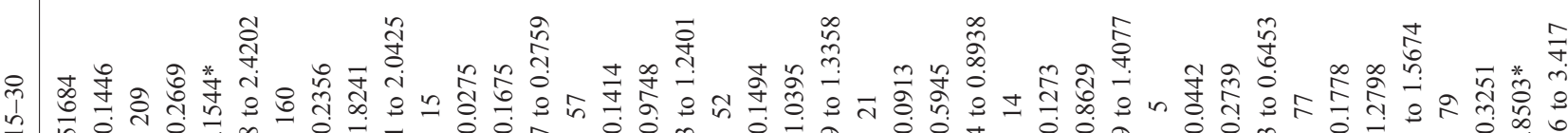

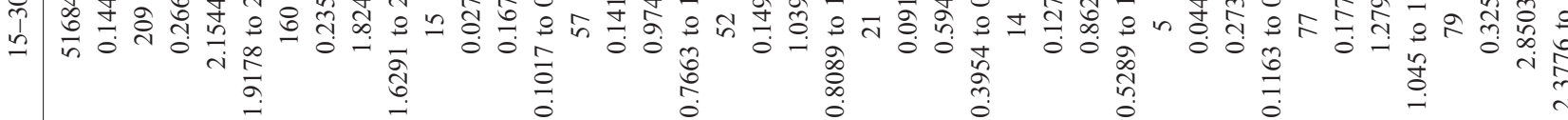

H

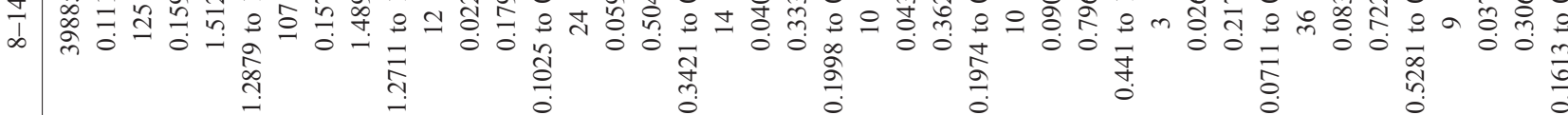

$\sim$ ح

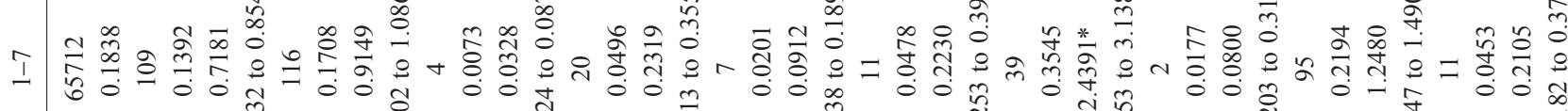

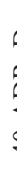

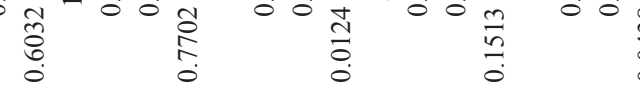

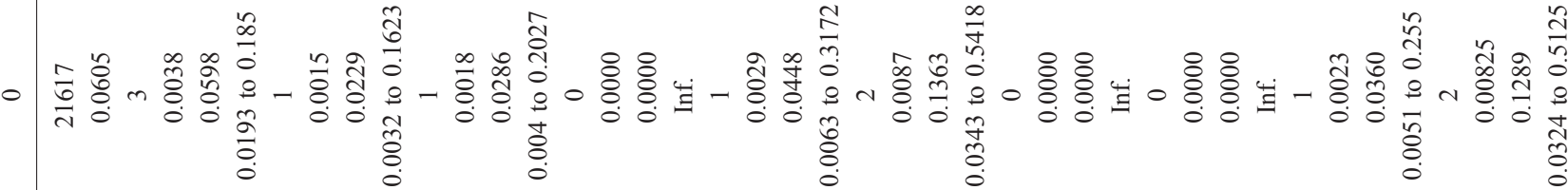

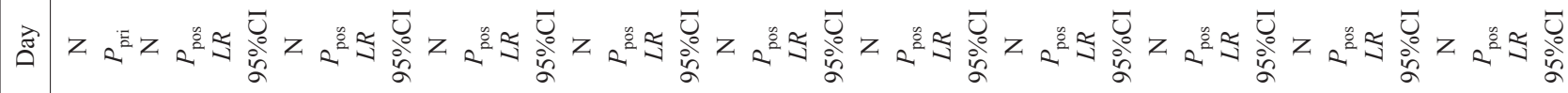
ํำ 节

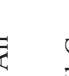
s

竞

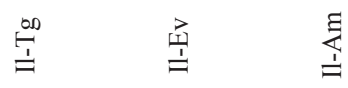

$\stackrel{9}{\stackrel{1}{ }}$

$\stackrel{\Xi}{!}$

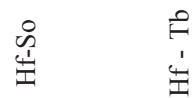




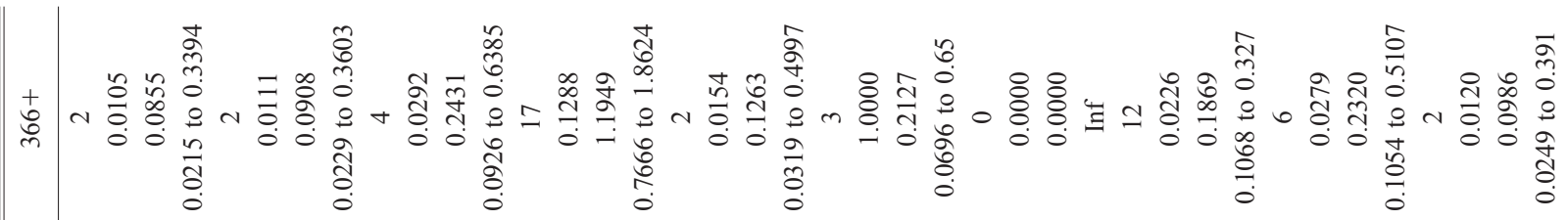

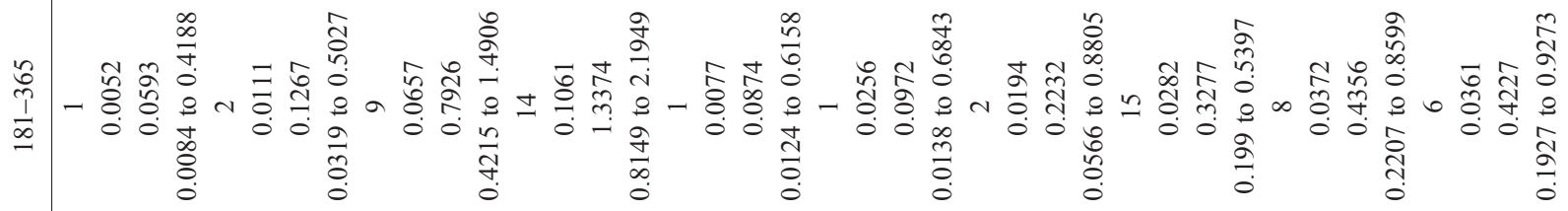

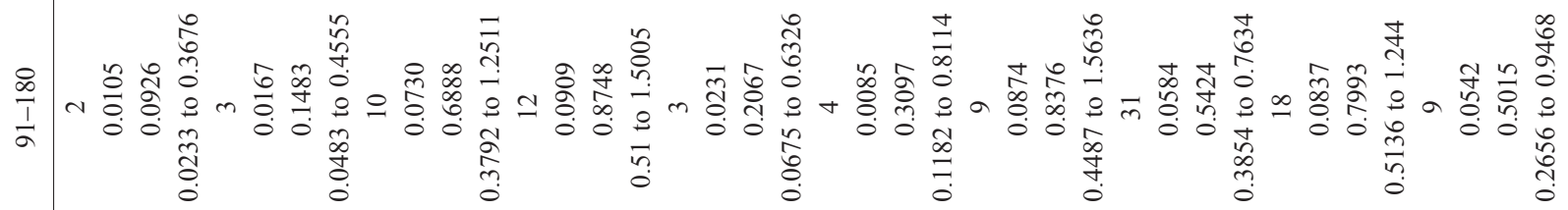

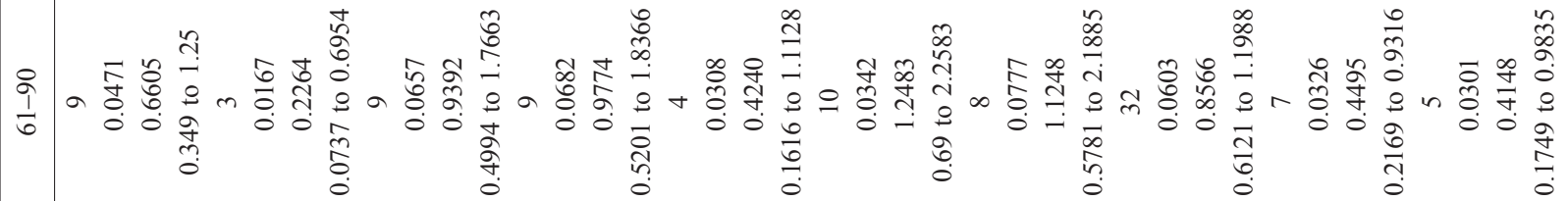

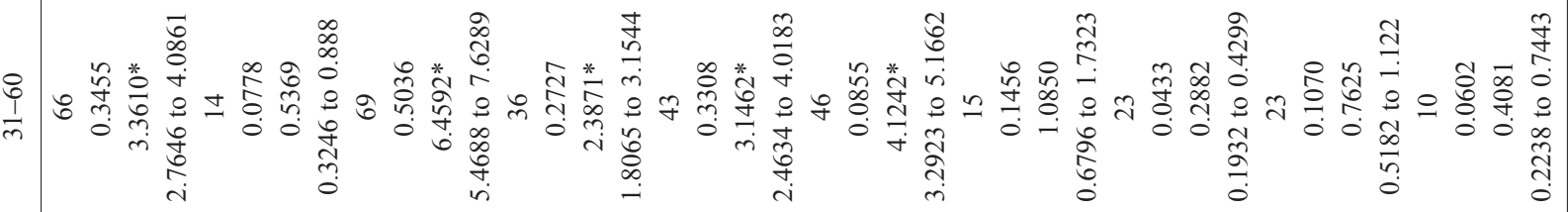

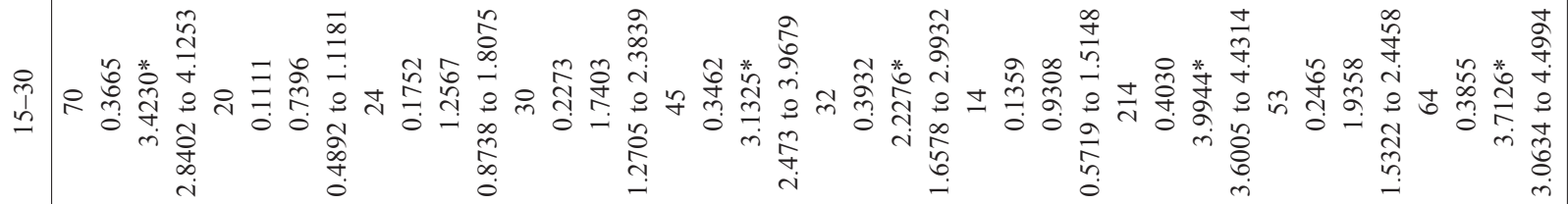

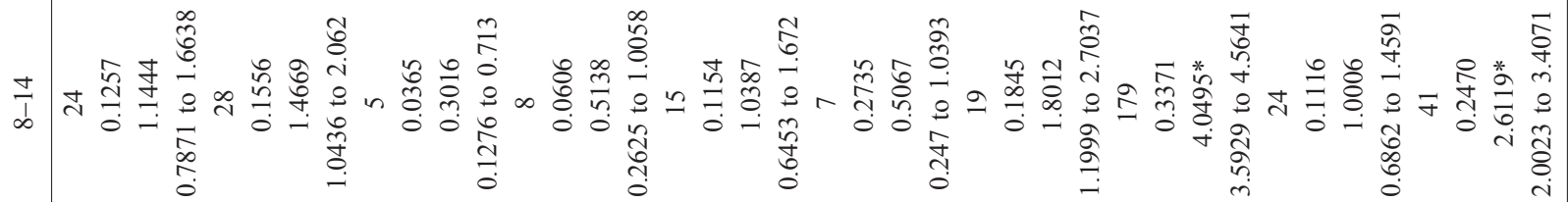

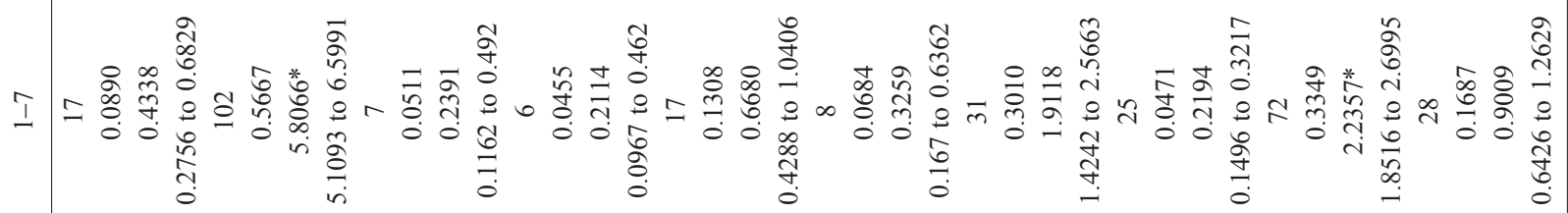

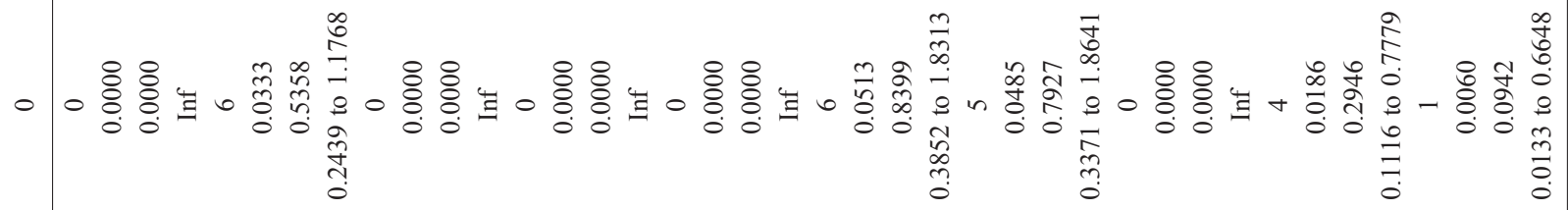

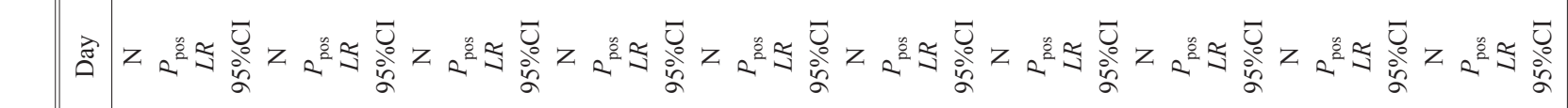

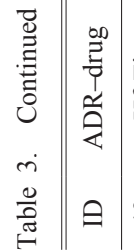

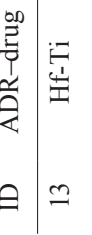

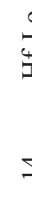
挡 应 崖 届

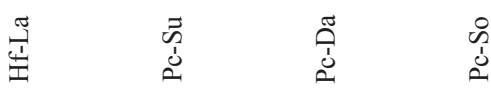
$\pm \quad$ $\cong \quad \infty$ 9 ำ ำ ง i 


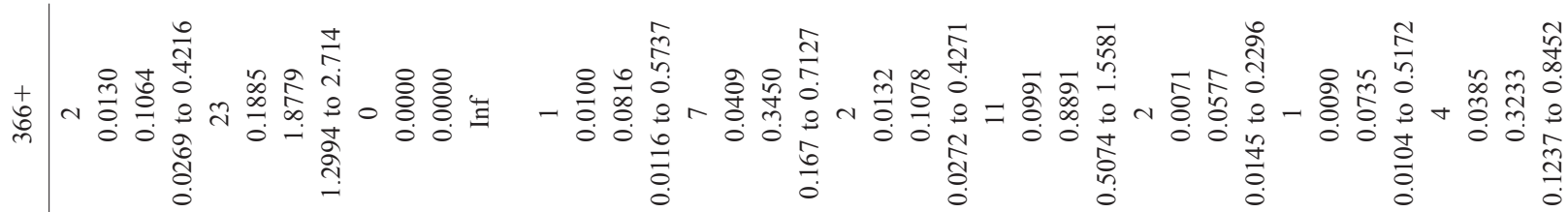

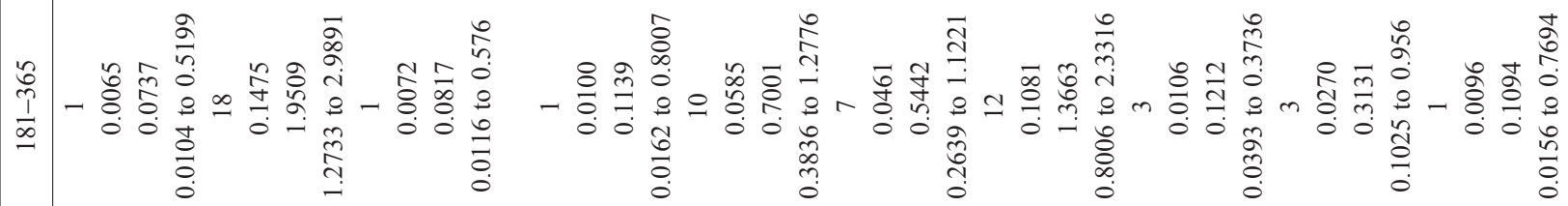

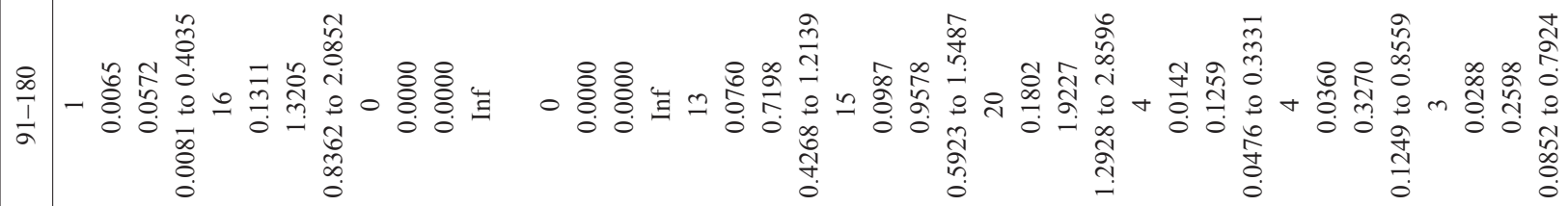

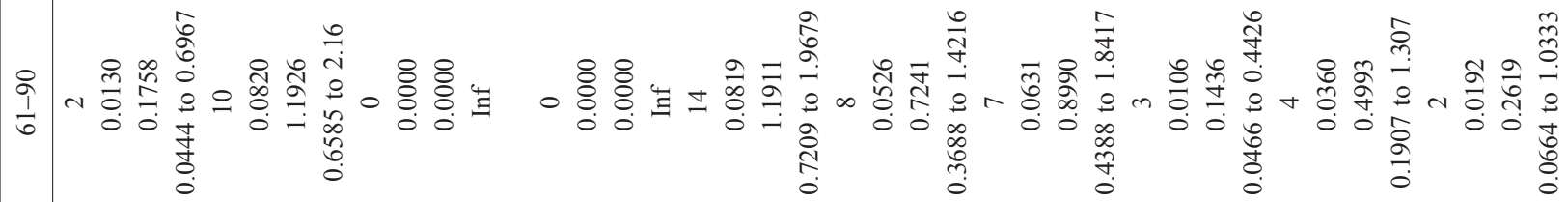

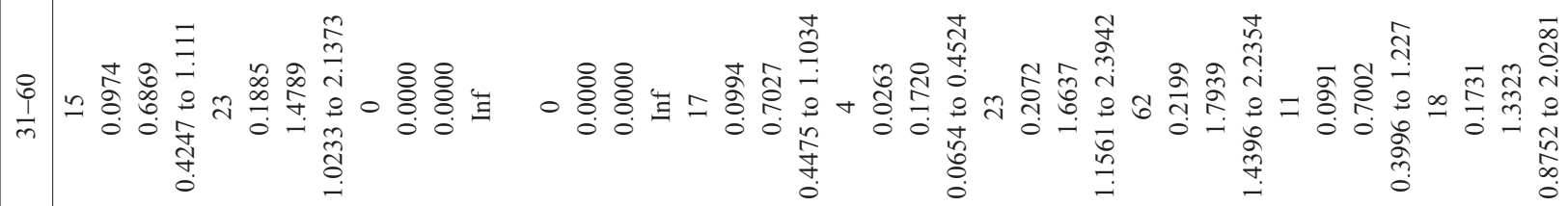

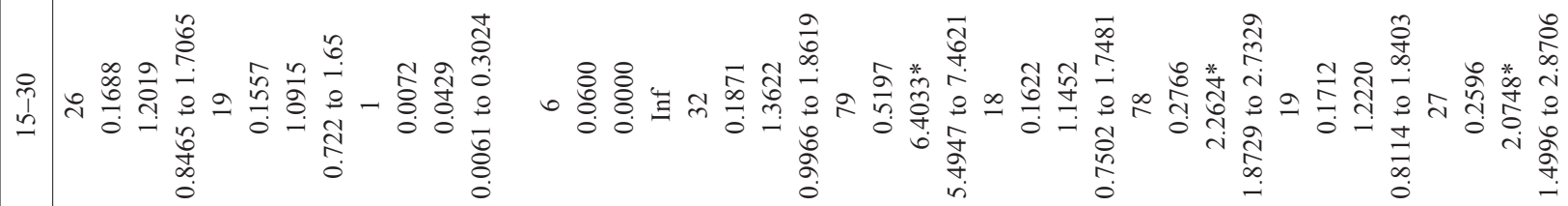

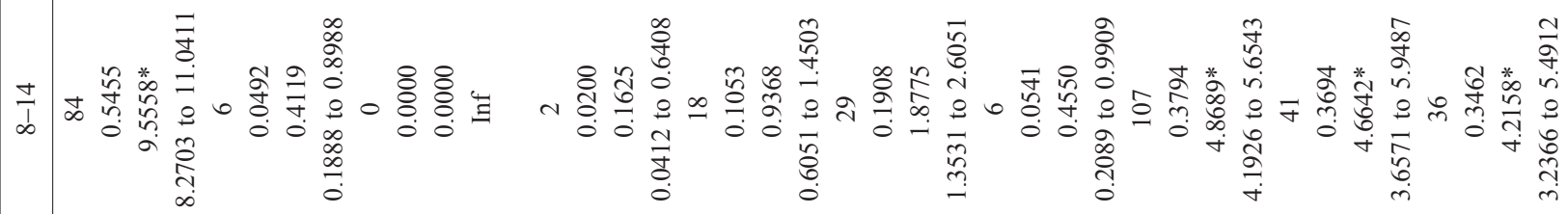

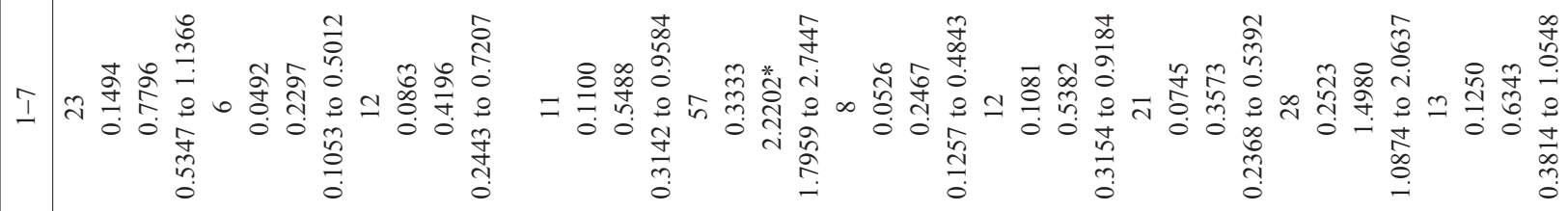

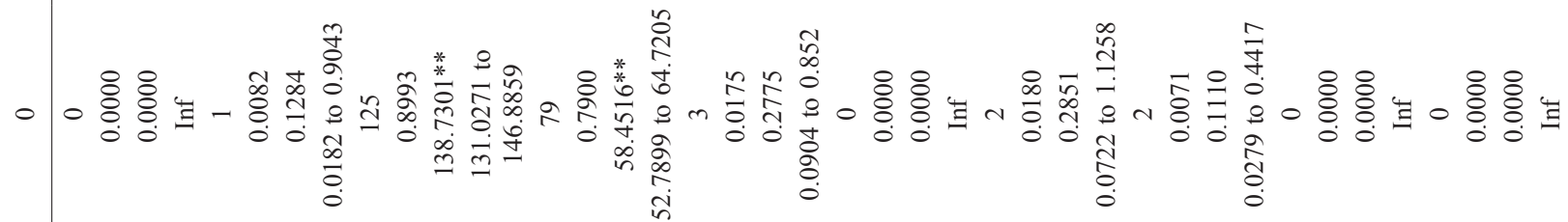

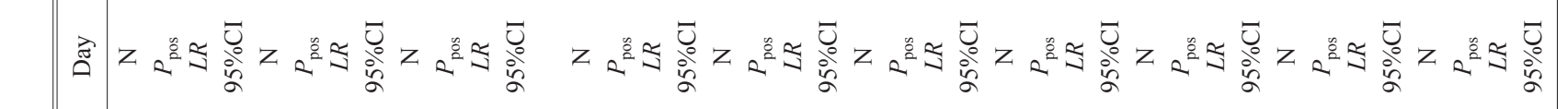

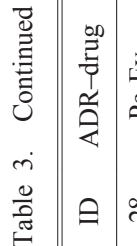
$\stackrel{n}{\stackrel{0}{1}}$

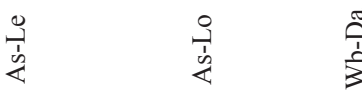

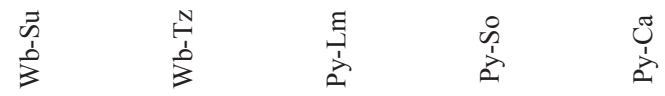
ㄹ के กี ช + f $\bar{n}$ $n$ in 


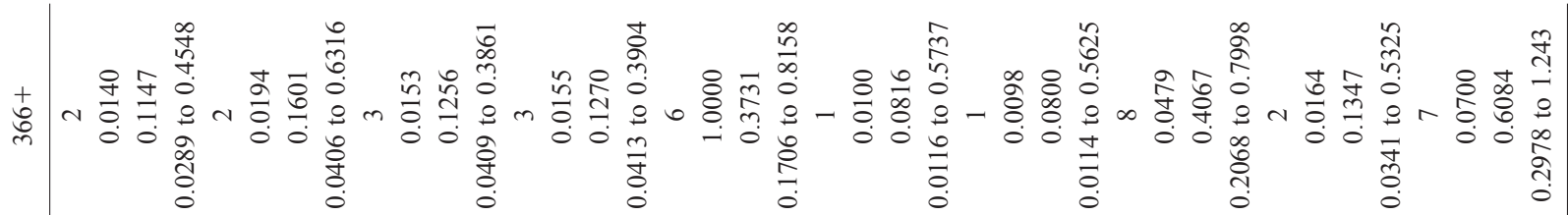

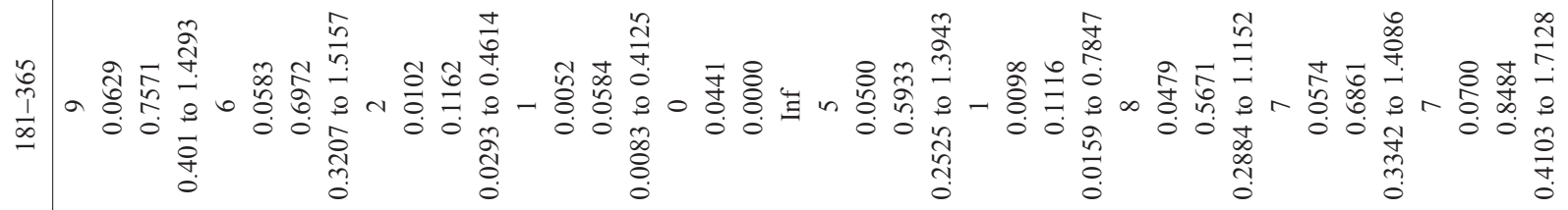

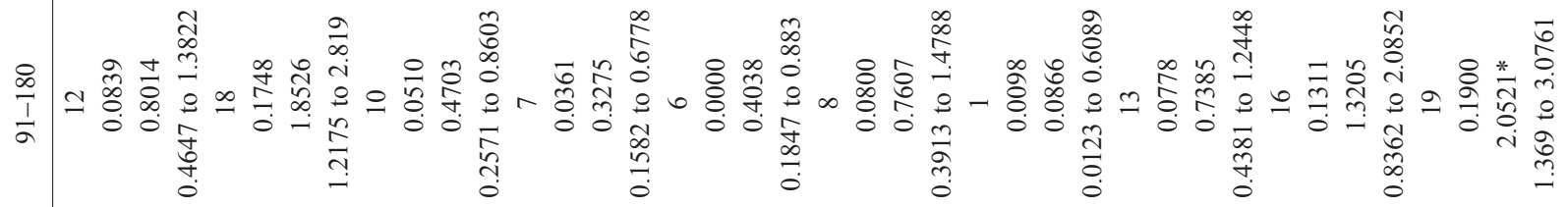

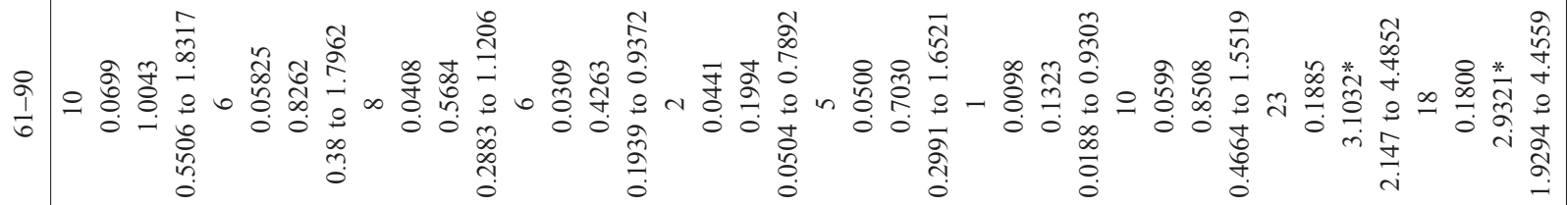

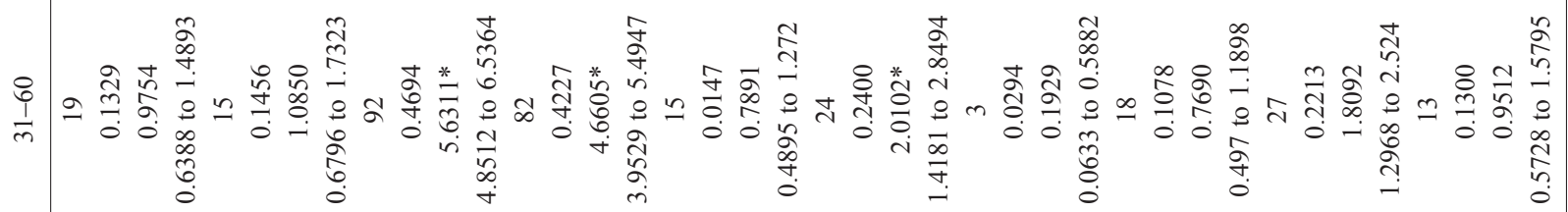

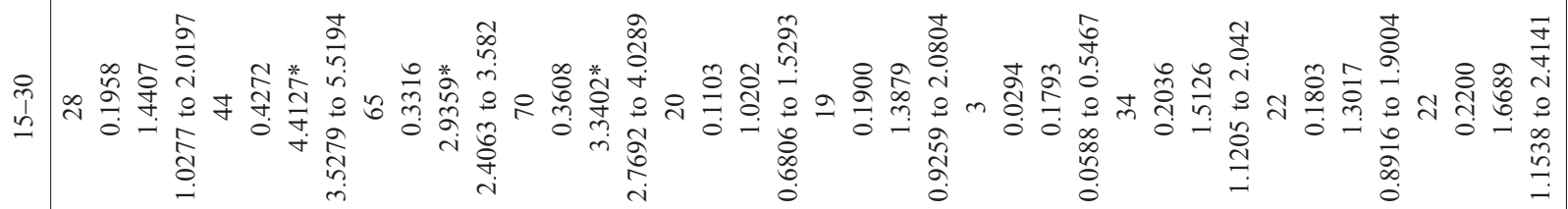

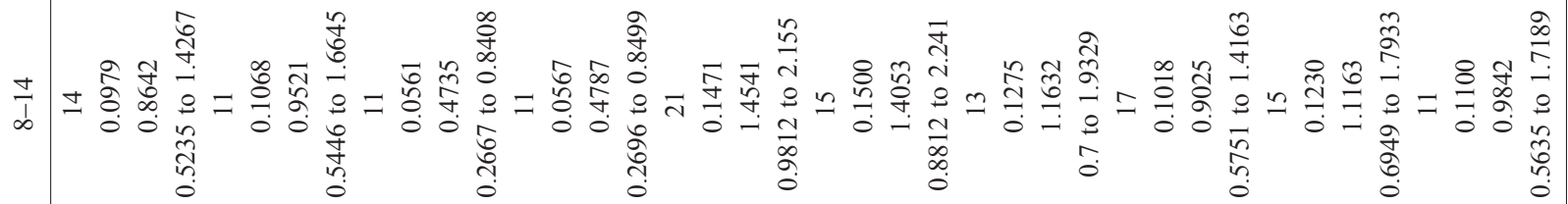
$\sim$ 击落

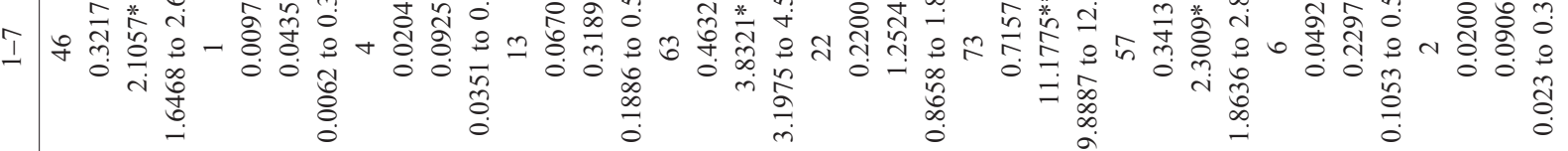

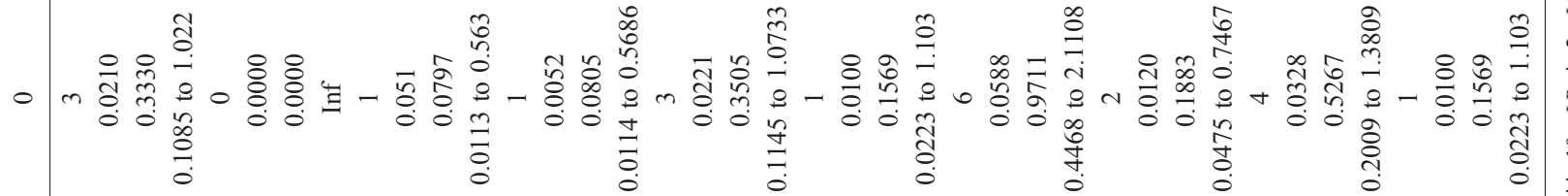

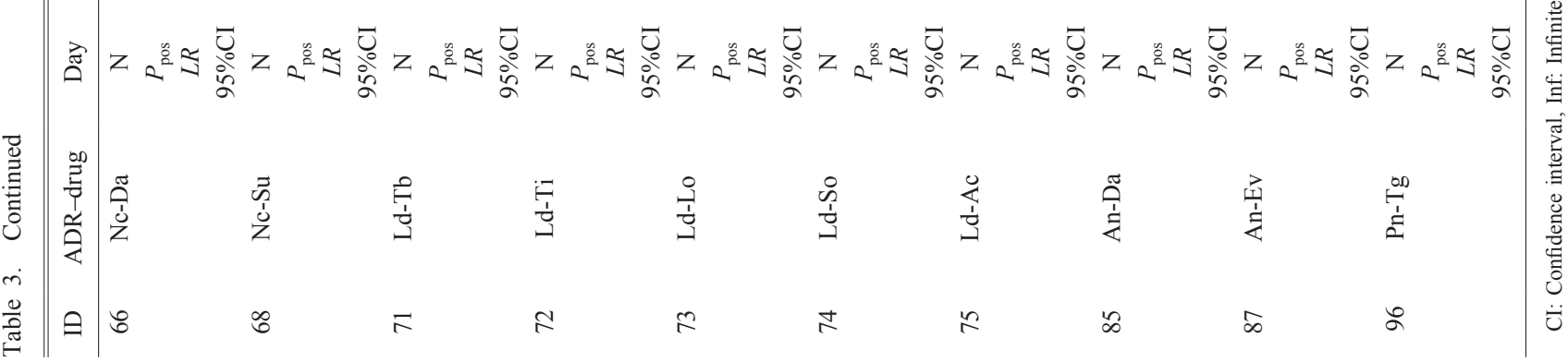


ADRs. ${ }^{716)}$ There were $68 \mathrm{~A}-\mathrm{D}-\mathrm{O}$ combinations with an $L R$ of $1-2$. Onset timing information for these A-D-O combinations is not likely to be clinically important. ${ }^{7,16)}$

There were $54 \mathrm{~A}-\mathrm{D}-\mathrm{O}$ combinations with an $L R$ greater than 2. In the case of these 54 combinations, onset timing information could be clinically useful. When we included the starting day of medication (i.e., $0 \mathrm{~d}$ ) as Nday-onset-info for the combination of anaphylactic shock with levofloxacin (Le) (ID 31 in Table 3) or loxoprofen (Lo) (ID 32 in Table 3), the $L R$ was 138.7301 or 58.4516. Similarly, when we included the Nday-onset-info (i.e., 1-7 d) for the combination of liver disorder with acetaminophen (Ac) (ID 75 in Table 3), the $L R$ was 11.1775. This indicates that for these three combinations, onset timing information is clinically very useful. ${ }^{16)}$

\section{CONCLUSION}

Using Bayes' theorem, we quantitatively evaluated the extent to which information on the days to onset of ADRs after taking drugs is clinically useful to patients in identifying ADRs. In the case of anaphylactic shock due to levofloxacin or loxoprofen administration, the results suggested that information on the number of days of using the drugs is extremely useful for patient awareness of the ADR. We also showed that for the combination of liver disorder and acetaminophen, the addition of onset timing information showed a high likelihood ratio. From the results of this study, the offering of ADR onset timing information to patients by pharmacists to raise patient awareness of ADRs, together with the recognition by pharmacists of characteristic onset timing information for ADRs with specific drugs, are clinically useful because patient monitoring of changes in their physical condition while taking medication can prevent ill effects associated with ADRs from becoming more serious.

Study Limitations The data analyzed in this study are held in a spontaneously reported adverse event database system. They are therefore subject to bias by under-reporting, and prone to the effects of the release of safety information by the regulatory authorities, and to market trends.

Conflict of Interest The authors declare no conflict of interest.

\section{REFERENCES}

1) Oshima $\mathrm{S}$, Senoo K, Negishi A, Akimoto H, Ohara K, Inoue $\mathrm{N}$, Ohshima S, Kutsuma N, Juni K, Kobayashi D. Identification of the discrepancies between pharmacist and patient perception of the pharmacist's role as an advisor on drug therapy based on social sci- ence theory. Biol. Pharm. Bull., 39, 313-322 (2016).

2) Tsuchiya F. Revision of Pharmaceutical Affairs Law/Pharmacist Law, and providing patients with information on adverse drug reactions. Yakkyoku, 49, 1976-1982 (1998).

3) Ohshima S, Ieda M, Yamamoto M, Kobayashi D. Quantitative evaluation method of information value of initial symptoms based on Bayesian theory. Yakugaku Zasshi, 132, 763-768 (2012).

4) Kobayashi D, Hosaka S, Inoue E, Ohshima K, Kutsuma N, Oshima $\mathrm{S}$, Okuno Y. Quantitative evaluation of initial symptoms as predictors to detect adverse drug reactions using Bayes' theory: expansion and evaluation of drug-adverse drug reaction-initial symptom combinations using adverse event reporting system database. Biol. Pharm. Bull., 36, 1891-1901 (2013).

5) Ghajar BM, Lanctôt KL, Shear NH, Naranjo CA. Bayesian differential diagnosis of a cutaneous reaction associated with the administration of sulfonamides. Semin. Dermatol., 8, 213-218 (1989).

6) Ikesue H. Development of work sheets to provide efficient pharmaceutical care during cancer chemotherapy and their evaluation. Jpn. J. Pharm. Health Care Sci., 32, 1-12 (2006).

7) Robert HF, Suzanne WF, Grant SF. Diagnosis. Clinical Epidemiology: The Essentials. (5th ed.), Lippincott Williams and Wilkins, a Wolters Kluwer business, Pennsylvania, pp. 123-124 (2012).

8) Hirooka T. "PMDA fukusayou database no katsuyou 3) fukusayou hatsugen madeno hatsugen pattern niyoru bunrui-nissuu data ni tsuiteno riyou.": 〈https://biostat.jp/archive_teireikai_2_download. php?id=44), cited 09 January, 2017.

9) Yamada M, Handa J. Comparison of the onset time profile among the interferon formulations in adverse drug reaction of suicide- or diabetes-related. Jpn. J. Pharmacoepidemiol., 19, 23-30 (2014).

10) Sauzet O, Carvajal A, Escudero A, Molokhia M, Cornelius VR. Illustration of the weibull shape parameter signal detection tool using electronic healthcare record data. Drug Saf., 36, 995-1006 (2013).

11) Nicholas PJ. Estimation and inference for measures of association. Statistics for Epidemiology. Chapman and Hall/CRC, Boca Raton, pp. 81-83 (2003).

12) Altman DG, Machin D, Bryant TN, Gardner MJ. Diagnostic tests. Statistics With Confidence. (2nd ed.), BMJ books, London, pp. 105-119 (2000).

13) Nishijima N, Azuma K, Araya S, Izawa K, Inoue K, Kusakari T, Jige H, Suga S, Suzuki Y, Takagi T, Takasugi Y, Murakami I, Watanabe M, Kawakami J, Seto K, Kitajima Y. Preliminary study on the need for data cleaning in PMDA's drug adverse event report database. Pharmaceutical and Medical Device Regulatory Science, 45, 616-622 (2014).

14) Umetsu R, Nishibata $Y$, Abe J, Suzuki Y, Hara H, Nagasawa H, Kinosada Y, Nakamura M. Evaluation of the association between the use of oral anti-hyperglycemic agents and hypoglycemia in Japan by data mining of the Japanese Adverse Drug Event Report (JADER) database. Yakugaku Zasshi, 134, 299-304 (2014).

15) R Core Team. "R: language and environment for statistical computing." R Foundation for Statistical Computing, 2005; Vienna, Austria. 〈http://www.R-project.org〉, accessed 15 Mar 2015.

16) Gallagher EJ. Clinical utility of likelihood ratios. Ann. Emerg. Med., 31, 391-397 (1998). 